http://jmscr.igmpublication.org/home/

ISSN (e)-2347-176x ISSN (p) 2455-0450

crossref DOI: https://dx.doi.org/10.18535/jmscr/v9i7.27

Journal Of Medical Science And Clinical Research

\title{
Carbonbaby Syndrome- Case Report of Two Siblings
}

Authors

Dr Manvi Katoch ${ }^{1}$, Dr Parmananda Roy ${ }^{2 *}$, Dr Shiwani Thakur ${ }^{3}$

${ }^{1}$ Anaesthesiologist, Zonal Hospital, Dharamshala, H.P.

${ }^{2,3}$ Senior resident, Department of Pediatrics, JLNMCH Bhagalpur, Bihar

*Corresponding Author

Dr Parmananda Roy

Senior Resident, Department of Pediatrics, JLNMCH Bhagalpur, Bihar, India

\begin{abstract}
Acquired universal melanosis also known as carbon baby syndromeis a rare condition of progressive generalised hyperpigmentation of skin and mucosal lining with histopathological features of increased epidermal basal layer melanisation. We diagnosed two siblings of the same family who developed progressive darkening of skin and mucosa since infancy with histopathological evidence of excessive melanin pigmentation of the epidermis.

Keywords: Acquired, generalised, excessive melanin pigmentation.
\end{abstract}

\section{Introduction}

Carbon baby syndrome is quite a rare skin pigmentation disorder where a child is born with normal skin colour but during infancy hyperpigmentation of skin and mucosal membrane develops. This abnormal discoloration progressive and generalised. We came across two such cases who were admitted in pediatrics department. Both the siblings were normal at birth but their skin started darkening during infancy.

\section{Case Report}

Case Report 1- An8-year old male child named Chotu, born of non-consanguineous marriage was admitted in the paediatrics department with complaints of progressive darkening of whole body. His mother had uneventful prenatal, natal and postnatal history. Initially, the pigmentation started in the face and hands at the age of 10 months which gradually, progressed over next 5 years with the involvement of whole body [Figure 1]. There was no history of fever, skin infection, darkening of urine, photosensitivity or any other systemic complaints. History of any prior drug intake was absent. He had positive family history of same disorder affecting his 3year old female sibling.

Case Report 2- A 3-year old female child named Kumkum, born of non-consanguineous marriage was also admitted to this department with the same complain of gradual and progressive hyperpigmentation of skin [Figure 1]. She was younger sibling of Chotu belonging to same family. Her mother had insignificant obstetrics history. Neonatal period was uneventful and the child had normal colour after birth. There was no history of any drug intake by the mother during pregnancy nor by the child during infancy. 
Gradually, during later infancy at 10 months of age, blackening of skin was noticed first on the face which subsequently spread to the whole body. There were no other skin lesions present on the body.

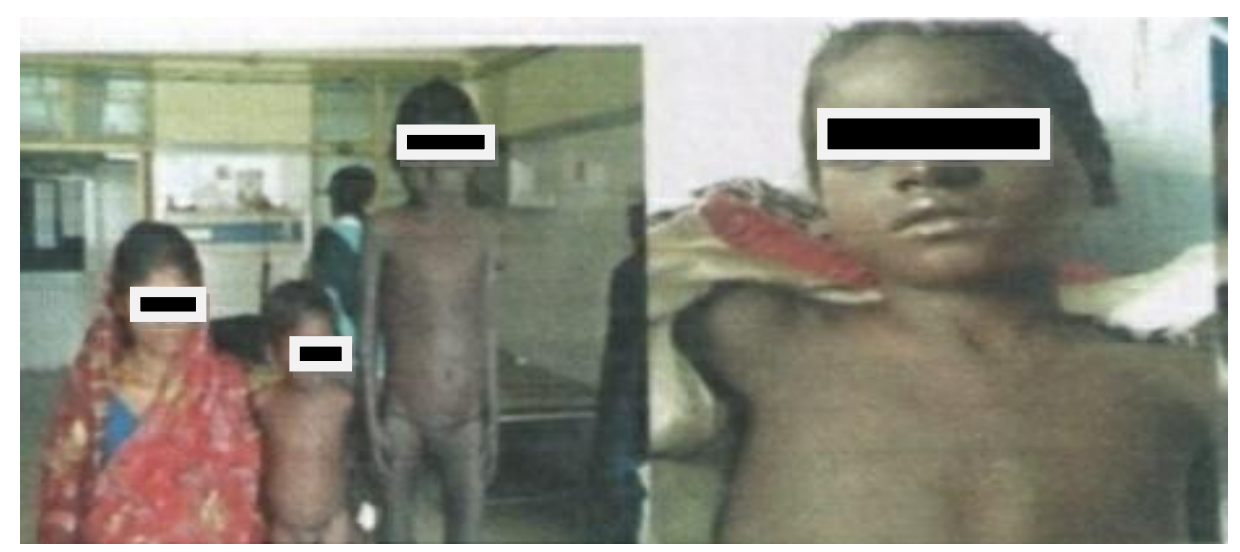

Figure 1 - Kumkum \& Chotu with diffused hyperpigmentation of whole body

On examination, both the children had dark black hyperpigmentation of whole body involving upper bulbar conjunctiva, oral mucosa and tongue also. Palms and soles were also affected [Figure 2]. Sun exposed areas had more pronounced skin changes but there were no changes in skin texture and sweat secretion. Both of them had normal growth and developmental parameters. Systemic and ophthalmological examination were within normal limits. Investigations were as follows- serum cortisol in male and female child was $11 \& 10$ microgram/dl in morning and 5.2 \& 3.3 microgram/dl at midnight respectively. Betamelanocyte stimulating hormone was $4.8 \& 5.5$ $\mathrm{pg} / \mathrm{ml}$ in male child and female child, respectively. ACTH levels were within normal limits. Routine blood and urine examination were also normal. Also, no abnormality was detected in LFT's and RFT's. Serum ferritin level and serum electrolytes were within normal range.

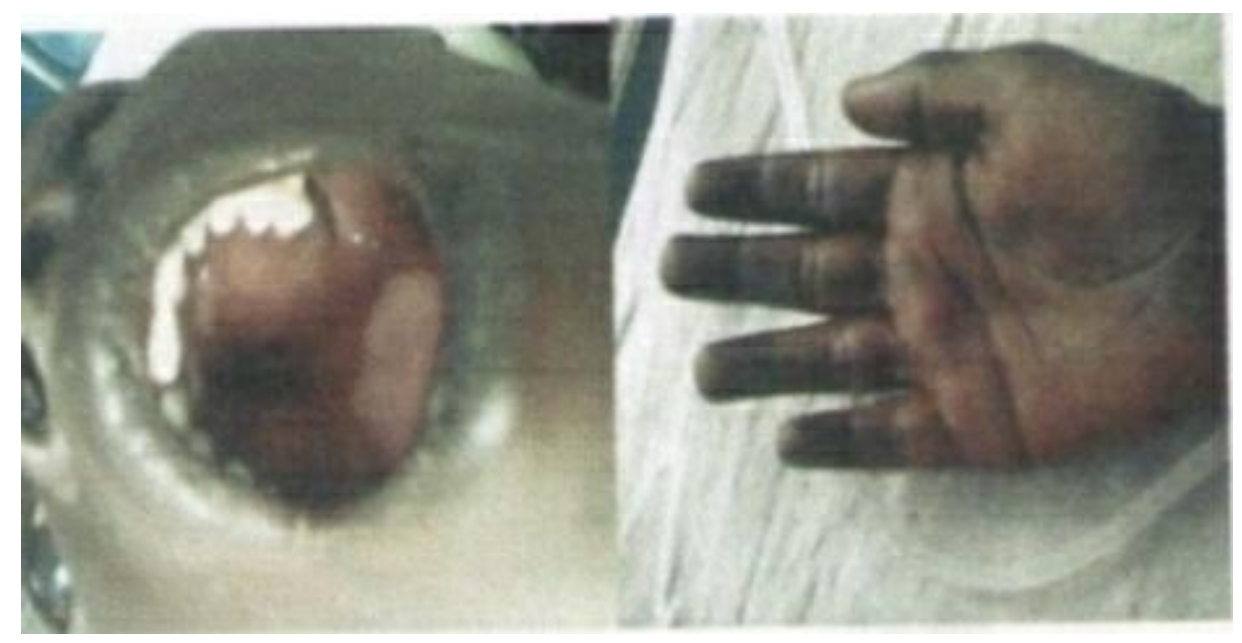

Figure 2 - Hyperpigmentation of lips and darkened palms

Skin biopsy report was remarkable which revealed coarse melanin laden melanocytes in the basal layer of entire epidermis without atypia [Figure 3,
Figure 4]. There was no increase in number of melanocytes. 


\section{JMSCR Vol||09||Issue||07||Page 151-154||July}

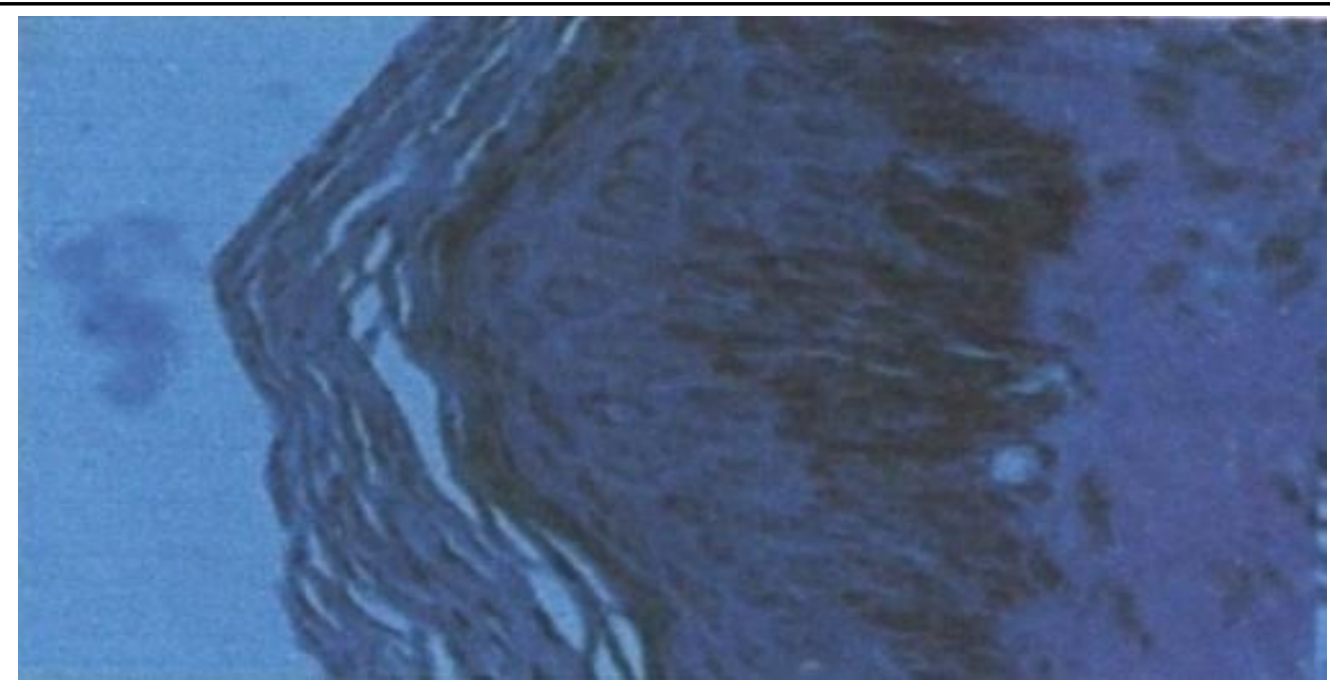

Figure 3 - Skin biopsy of Chotu showing increased pigmentation in basal layer of epidermis

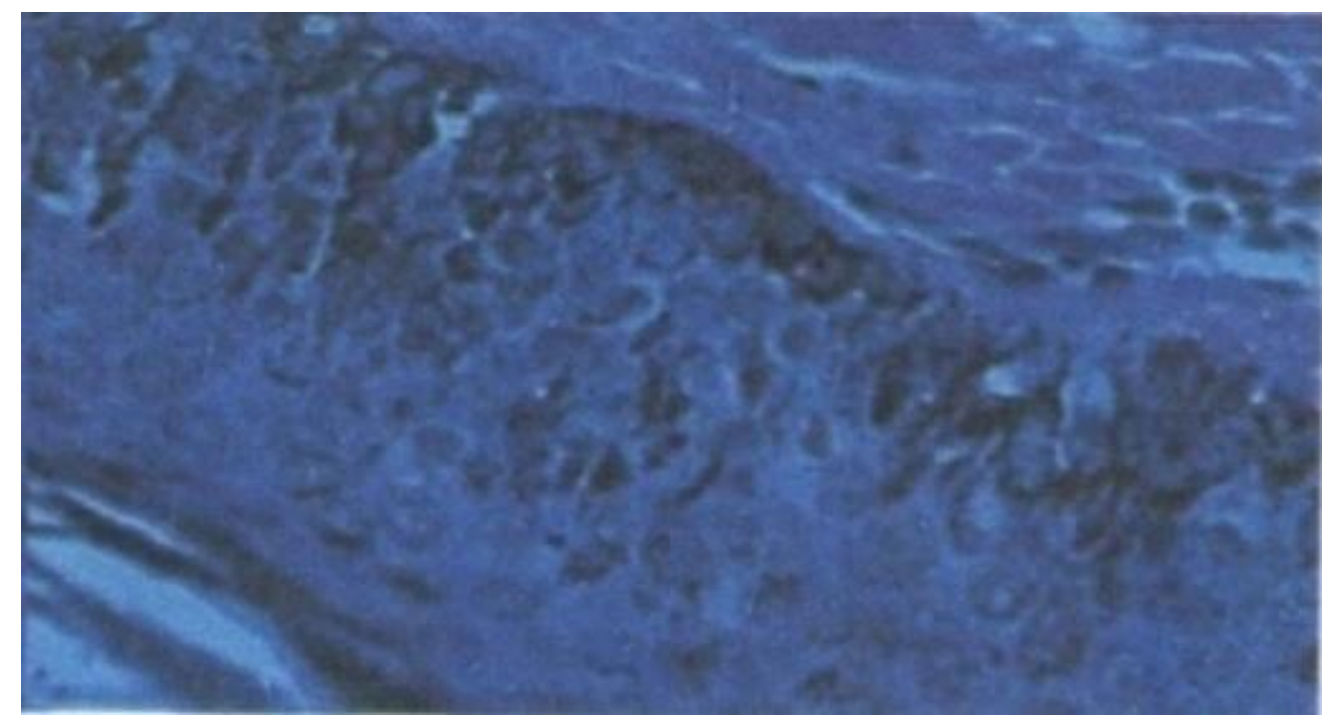

Figure 4 - Skin biopsy of Kumkum reveals similar increased pigmentation in basal layer of epidermis

\section{Discussion}

Among the various causes of diffuse hyperpigmentation of skin developing during infancy, classification is made on clinical and histological examination.

Acquired universal melanosis is an unusual condition of hyperpigmentation of skin and mucosa in which diagnosis is based on the principle of exclusion. There are many other skin conditions where pigmentation is not as generalised as seen in our case.

Cases of carbon baby syndrome have earlier been reported by Kaviarsan et al and Ruiz Maldonado where infants developed progressive skin darkening since infancy and histopathology showed increased melanin pigmentation in basal epidermal layer without increase in the number of melanocytes. ${ }^{1,2}$

Familial progressive hyperpigmentation is condition in which abnormal hyperpigmented skin patches are present since birth which gradually increase in size and number along with involvement of mucosal membranes. ${ }^{3}$ Histologically, melanocytes contain numerous large melanin granules since the time of birth unlike our case.

Two cases of congenital diffuse melanosis have been reported by Kint et al where skin hyperpigmentation started soon after birth progressing to involve the trunk and limb. ${ }^{4}$ The electron microscopy showed that melanosomes were not grouped with keratinocytes; instead 
melanocytes were dispersed throughout the cytoplasm of epidermal cells.

There is another condition of hyperpigmentation seen in neonates called bronze baby syndrome occurring due to hepatocellular dysfunction in babies undergoing phototherapy. The skin acquires grey brown discoloration due to photodestruction of porphyrin compound ${ }^{5}$, which was not found in our case. In adrenoleukodystrophy, which is an X- linked disorder, generalised hyperpigmentation of the skin occurs along with degeneration of brain and adrenal gland dysfunction. ${ }^{6}$ There is accumulation of 24-30 carbon unsaturated fatty acid in adrenal cortex and sphingolipids in the brain. Thus, this condition was also ruled out.

Hemochromatosis is characterized by generalized tanning of skin due to progressive iron deposition. This condition is easily differentiated by estimating serum ferritin level in blood and human hemochromatosis protein analysis, which were not observed in our cases. Among drugs, antimalarial, tetracycline, clofazimine, and heavy metal like gold, silver, bismuth and mercury are the important ones.

Diagnosis of our 2 cases of acquired universal melanosis is one of exclusion. We ruled out all the probable differential causes one by one and ultimately, skin biopsy clinched the diagnosis. Etiology of melanosis is still not clear. However, it has been proposed that there is increased sensitivity of melanocytes and over production of melanocyte stimulating hormone which leads to increased melanin production. However, 2 siblings of same family being affected also lead towards some genetic probability which needs more exploration.

\section{References}

1. Ruiz-Maldonado R, Tamayo L, FernandezDiaz J. Universal acquired melanosis: The carbon baby. Arch Dermatol.1978; 114:775-8.
2. Kaviarasan PK, Prasad PVS, Joe JM et al. Universal acquired melanosis (carbon baby). Indian J Dermatol Venereol Leprol 2008;74:38-40.

3. Chernosky ME, Anderson DE, Chang JP et al. Familial progressive hyperpigmentation. Arch Dermatol. 1971;103:581-2.

4. Kint A, Oomen C, Geerts ML, Breuillard F. Congenital diffuse melanosis. Ann Dermatol Venereol1987;114:11-6.

5. Ashley JR, Littles G, Reddi E. Bronze baby syndrome: A new porphyrin related disorder. Pediatr Res 1983;17:327-30.

6. Moser HW, Mahmood A, Raymond GV. X-linked adrenoleukodystrophy. Nat Clin PractNeurol2007;3:140-51. 